Technical notes

\title{
A scheme for assessing the performance characteristics of small field- of-view gamma cameras
}

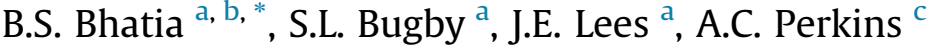 \\ a Space Research Centre, Physics \& Astronomy, University of Leicester, Leicester LE1 7RH, UK \\ b Imaging Clinical Group, Sandwell and West Birmingham NHS Hospital Trust, West Bromwich B71 4HJ, UK \\ ${ }^{\mathrm{c}}$ Medical Physics and Medical Imaging Unit, School of Medicine, University of Nottingham, Nottingham NG7 2UH, UK
}

\section{A R T I C L E I N F O}

\section{Article history:}

Received 16 January 2014

Received in revised form

13 August 2014

Accepted 20 August 2014

Available online 13 November 2014

\section{Keywords:}

Small field-of-view gamma camera

Performance testing

Performance characteristics

\begin{abstract}
A B S T R A C T
Existing protocols for assessing the performance characteristics of large field-of-view (LFOV) gamma cameras can be inappropriate and require modification for use with small field-of-view (SFOV) gamma camera systems. This communication proposes a generic scheme suitable for evaluating the performance characteristics of SFOV gamma cameras, based on modifications to the standard procedures of NEMA NU1-2007. Key differences in methodology between tests for LFOV and SFOV gamma cameras are highlighted along with the rationale for these changes. It is envisaged that this scheme will provide more appropriate methods for equipment characterisation, ensuring quality and consistency for all SFOV cameras.
\end{abstract}

() 2014 Associazione Italiana di Fisica Medica. Published by Elsevier Ltd. This is an open access article under the CC BY license (http://creativecommons.org/licenses/by/3.0/).

\section{Introduction}

Gamma cameras have traditionally been large devices comprising of inorganic scintillators, photomultiplier tubes, lead collimators and bulky lead shielding. These devices are generally not portable and so cannot be used for intraoperative procedures.

The development of smaller portable gamma cameras has enabled imaging procedures to be undertaken at the bedside, in intensive care units, clinics and in the operating theatre [1,2]. Highresolution small field-of-view (SFOV) gamma cameras have been designed for specific applications such as tumour resection and sentinel node localisation [3,4]. These aim to combine the advantages of large field-of-view (LFOV) gamma cameras and nonimaging gamma probes while addressing some of the limitations of these devices. Designs for SFOV systems include: scintillationbased detectors - with scintillators coupled to position sensitive photomultiplier tubes (PSPMTs) [5-8], electron multiplying charge coupled detectors (EMCCDs) $[9,10]$ or silicon drift arrays [11] - and solid state detectors such as cadmium telluride (CdTe) [12-14] and cadmium zinc telluride (CdZnTe) [15].

Manufacturers of LFOV gamma cameras routinely use standardised protocols such as the NEMA Standard NU1-2007 [16] to assess performance and provide specifications. In the clinical

\footnotetext{
* Corresponding author. Space Research Centre, Physics \& Astronomy, University of Leicester, Leicester LE1 7RH, UK. Tel.: +44 1162525519.

E-mail address: bsb13@le.ac.uk (B.S. Bhatia).
}

environment, modified protocols arising from these standards have been developed for ease of use, for example, IPEM Report 86 in the UK [17]. The European Directive 97/43/EURATOM mandates a quality assurance programme for all medical devices used in diagnostic radiology, nuclear medicine and radiotherapy [18]. Routine quality control recommendations for LFOV gamma cameras and handheld gamma probes are well documented by the European Association of Nuclear Medicine (EANM) [19,20]. While LFOV gamma cameras are routinely tested using these standardised protocols $[16,17,21]$ such protocols are not always appropriate for or easily translated to SFOV gamma cameras. This communication proposes updated procedures for evaluating the performance characteristics of SFOV gamma cameras based on modifications to the NEMA NU1-2007 standard [16].

\section{Rationale}

This section outlines the current standard characterisation approach used for LFOV systems and any modifications necessary for assessing SFOV systems. The assessed parameters are spatial resolution, spatial distortion, spatial uniformity, count-rate capability, sensitivity, and energy resolution.

The duration of imaging for all tests should be sufficient to limit the effects of statistical noise. In line with LFOV measurements, a minimum of 150 counts per pixel should be collected, with higher counts per pixel when only a small area of the detector is irradiated 
[16]. Additional performance tests for collimator performance and shield leakage are discussed elsewhere [19,21].

\section{Intrinsic spatial resolution}

This is defined as the full-width at half-maximum (FWHM) of a line spread function (LSF) or of a point spread function (PSF) without an imaging collimator installed. This measurement should be supplemented by the full-width at tenth-maximum (FWTM) as the PSF or LSF may deviate from a Gaussian profile.

Standard methodologies for LFOV gamma cameras $[16,17,19,21]$ use a capillary line source of approximately 40MBq activity, of internal diameter of $0.5 \mathrm{~mm}$. This is positioned parallel to the principal orthogonal axes of the camera to avoid broadening of the LSF. The source is placed directly on top of the uncollimated scintillator crystal.

The intrinsic resolution of a LFOV gamma camera is typically in the region of $3 \mathrm{~mm}$ [22]. If an imaging matrix of $256 \times 256$ pixels is used, the pixel dimension of a $540 \mathrm{~mm}$ diameter gamma camera (to choose a single example) will be around $2.1 \mathrm{~mm}$. NEMA NU1-2007 [16] states the "pixel size should be less than or equal to 0.1 FWHM", that is $\leq 0.3 \mathrm{~mm}$ for a $540 \mathrm{~mm}$ diameter gamma camera. To achieve the specified "pixel size" the analogue to digital conversion gain is increased perpendicular to the line source for each orthogonal axis simultaneously, and the "zoomed" portion of the fieldofview is imaged.

SFOV cameras have reported values of spatial resolution of less than $1.0 \mathrm{~mm}[5,9-11,14]$, suggesting that the NEMA "pixel size" should be at most $0.1 \mathrm{~mm}$ (equal to 0.1 FWHM).

For a typical LFOV resolution of $3 \mathrm{~mm}$, the use of a $0.5 \mathrm{~mm}$ line source will not have a large effect on measured resolution; at submillimetre resolution, however, the width of the same source becomes significant. Following the standards for LFOV systems, line source width (or the diameter of the point source) would need to have dimensions less than $0.1 \mathrm{~mm}$ The uniform filling of capillary tubes with diameters of the order $100 \mu \mathrm{m}$ is difficult to achieve. On this scale even the manufacture of a slit transmission phantom becomes challenging. This method of intrinsic spatial resolution measurement is therefore not suitable for high-resolution SFOV systems.

An alternative derivation of the FWHM can be obtained using the edge response function (ERF) method [16,23]. This can be obtained using a mask with a machined edge. When irradiated with a uniform radioactive source such that incident gamma photons can be assumed to be perpendicular to the mask plane, the detected counts across the edge of the mask ideally correspond to a step function, the derivative of which gives a LSF [23] which may then be analysed as in LFOV protocols.

\section{System spatial resolution}

This is defined as the FWHM of a LSF or of a PSF with the imaging collimator in place.

The protocol for LFOV gamma cameras uses a capillary line source (internal diameter less than $0.5 \mathrm{~mm}$ ) with FWHM response measured in air and with scattering media (such as Perspex) positioned between the line source and the collimator surface [16]. The Perspex acts to scatter photons as would be expected from a source inside a patient. Typically, LFOV system resolution measurements are stated in the context of the collimator used either at the collimator face or at a known distance (usually $100 \mathrm{~mm}$ ) away from the collimator. System resolution is typically limited by the type of collimator used rather than the intrinsic resolution of the detector.
Similar to intrinsic resolution measurements, for SFOV cameras the line source width or point source diameter would ideally be smaller than that used for LFOV measurements, again proving difficult to manufacture and fill [24]. The benefit of a consistent approach across all gamma cameras outweighs the effects of a finite source and the standard LFOV method, with a $0.5 \mathrm{~mm}$ diameter line source, may be used.

It may be possible to use a point or line source of a known diameter and then deconvolve the expected profile from the resultant image to determine the resolution; this is not ideal and requires specific knowledge of the expected profile of the source [10] and so may produce inconsistent results for different systems.

Many SFOV cameras use pinhole collimators rather than the more widely used parallel-hole collimator. This means that a line source imaged at the collimator face would appear to be a flood source in the resultant image. Instead of reporting resolution at the collimator face, measurements for pinhole systems should be stated at the non-magnifying point. As resolution varies significantly with aperture to source distance (through scattering material), the relationship between these two factors should also be reported so that resolution may be calculated by the end user for any source distance.

\section{Intrinsic spatial distortion}

Spatial distortion is a measure of how accurately event positions are mapped to the resulting image. For LFOV gamma cameras, spatial non-linearity is assessed using a lead transmission mask with an uncollimated detector [16]. A least-squares fit for the imaged line position is calculated. The differences between the imaged and fitted lines at $10 \mathrm{~mm}$ intervals are obtained to specify the spatial non-linearity differences across the geometric field-ofview (GFOV). In this paper, the GFOV is the whole non-magnified fieldofview of the gamma photon detector. Reported values are the standard deviation, mean and maximal difference between the imaged and fitted lines.

For LFOV cameras the mask used is a parallel line equal spacing (PLES) phantom, which consists of a series of parallel $1 \mathrm{~mm}$ wide lead lines spaced at $20 \mathrm{~mm}$ apart, embedded in uniform grooves within Perspex. A PLES phantom scaled to a $\sim 40 \mathrm{~mm}$ fieldofview would require precise manufacturing.

With smaller fields of view, spatial distortion in SFOV cameras can be measured with a line source at a range of orientations. Where several measurements are required to cover the FOV, multiple images can be acquired.

\section{Intrinsic spatial uniformity}

Spatial uniformity describes the variation in counts per pixel within the GFOV relative to the mean counts per pixel over the field of view. Intrinsic measurements are performed with the collimator removed. A point source at a distance of at least five times the useful field-of-view (UFOV) away from the crystal is used to irradiate the detector uniformly - this method can translate directly to SFOV systems. The UFOV is the collimated field-of-view of the gamma camera.

Uniformity should be reported with both an integral (across the entire detector) and differential (for localised groups of pixels) parameter. The typical measure for integral uniformity (IU) is calculated, as Equation (1) [17] where $C$ indicates number of detected counts per pixel in the image. Differential uniformity may be calculated using Equation (1) for small groups of adjacent pixels. Across the whole image differential uniformity values can be combined to a single reporting parameter [17], as described later in Sections analysis and reporting parameters. 
$\mathrm{IU}=\left(\frac{C_{\max }-C_{\min }}{C_{\max }+C_{\min }}\right) \times 100 \%$

IU is the integral uniformity; $C_{\max }$ and $C_{\min }$ are respectively the maximum and minimum number of detected counts per pixel in the image.

This method, however, is not robust when outlying pixel values are present. IPEM Report 86 [17] suggests reporting the coefficient of variation (standard deviation of counts per pixel to the mean counts) for integral uniformity. These parameters are suggested as standard for SFOV measurements to maintain consistency and to avoid unrepresentative values caused by unusually high or low outliers. For SFOV cameras using collimators that may introduce non-uniformities (such as pinhole collimators), a system spatial uniformity should also be reported.

\section{Count-rate capability}

Count-rate capability is characterised by the count-rate response curve showing the measured count-rate versus expected count-rate from a radioactive source. Among other factors, the count-rate response curve depends on the energy spectrum of the detected photons, and so depends on the amount of scatter present [16]. The range of expected count-rates should at least correspond to activities typically used clinically. A count-rate response curve can be plotted by allowing the source(s) to decay and recording measured count-rate at known time intervals. The same basic procedure can be used for SFOV cameras with the source size, position and activity matched to the characteristics of the type of camera being tested.

\section{System sensitivity}

Sensitivity is defined as the proportion of photon flux incident on the detector that is recorded within the photopeak energy window being used. The type of collimator should be specified when stating the camera system sensitivity.

For both LFOV and SFOV gamma cameras, a uniform planar source covering the GFOV at a known distance away from the camera face should be used. Incident counts at the detector can be calculated taking into account the initial activity, distance, time of image and solid angle subtended by the detector.

Pinhole collimators are likely to be used with SFOV gamma cameras so as to increase the field of view. As sensitivity is known to vary with distance away from the pinhole collimator face [25], system sensitivity measurements should be performed with up to $100 \mathrm{~mm}$ of scattering medium to ensure that clinical imaging does not miss deep-seated lesions [26]. These measurements should be repeated without scattering medium between the source and the detector.

Intrinsic sensitivity, without a collimator in place, should also be reported for all SFOV systems, as this value may vary over time for some detector types.

\section{Energy resolution}

Energy resolution is defined as the FWHM of the photopeak of the principal emission energy of the radionuclide being imaged. The energy spectrum is accumulated using a point source at a distance of at least five times the longest dimension of the UFOV, fixed centrally above the camera face. Measurements should be performed using different radionuclides covering the clinically useful energy range, and repeated to ensure the camera is stable with respect to drift of the detected photopeak energy. SFOV cameras may follow LFOV protocols without adjustment for this parameter.

\section{Scheme}

This section outlines a complete scheme for assessing the performance characteristics of SFOV cameras and may be used in part to investigate a particular parameter or as a whole to provide a complete characterisation.

For each test, activity should be chosen so as to achieve an appropriate number of counts within the count-rate capability of the detector. Unless otherwise stated, uniformity and noise corrections specific to the gamma camera under test and energy windowing should be performed as standard for clinical use. The energy window used for testing (as a percentage width across the photopeak) should be reported.

\section{Intrinsic spatial resolution}

\section{Method}

A mask with a machined edge, manufactured from a material with low transmission for the gamma photon energies being used, is required. The edge should be perpendicular to the mask surface and straight to an accuracy of at least $10 \%$ of the expected spatial resolution. The mask thickness should at a minimum be sufficient to attenuate $99 \%$ of photons, although a thicker mask (e.g. $20 \mathrm{~mm}$ ) would be preferable to exclude divergent photons. A point source (3 mm diameter maximum) or uniform flood source is also required.

The mask is placed as close to the scintillation crystal as camera design allows. The edge of the machined mask or internal edges of the line slit transmission mask should be positioned parallel to the orthogonal axes of the detector array.

The flood source, or point source at a distance of at least 100 times its diameter (to ensure uniformity), irradiates the mask. The

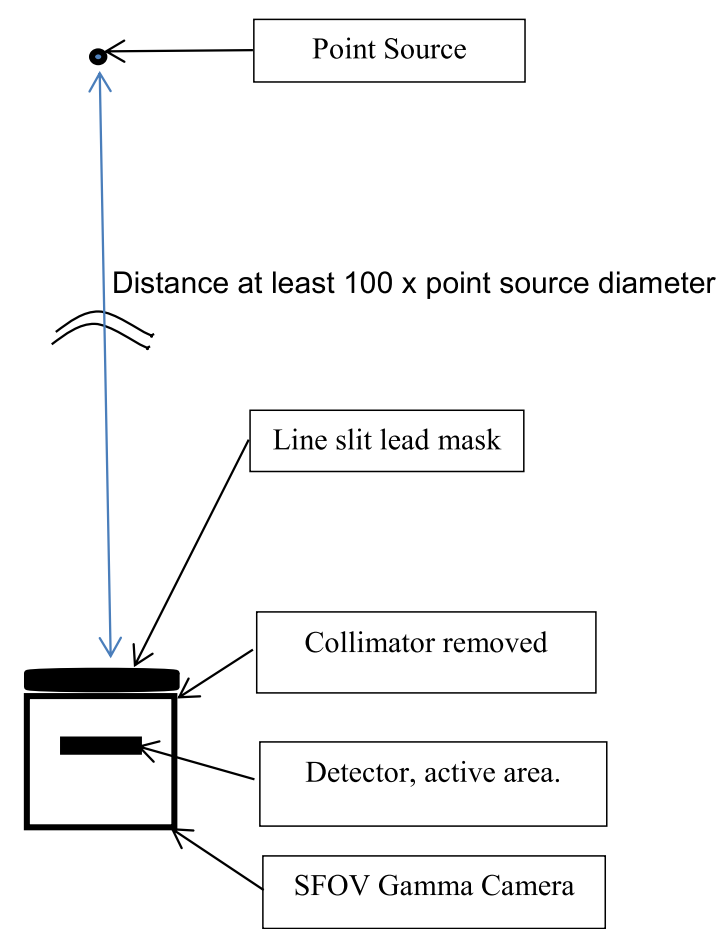

Figure 1. Intrinsic spatial resolution experimental setup. 
point source should be perpendicular to the camera face and in line with its centre, Fig. 1.

Measurements should be taken without any scattering media. A measurement is required in alignment with both the $x$ - and $y$-axes of the detector array.

\section{Analysis}

During processing an ERF is calculated; this is a plot of distance from the edge against normalised signal. If the edge is not perfectly aligned with the camera axes, this must be taken into account when creating the ERF. In this case a line of best fit is calculated for edge position and the distance from the calculated edge used in the ERF.

The ERF is then differentiated to give a LSF.

This should be repeated for the second orientation of the mask.

\section{Reporting parameters}

Intrinsic resolution is to be reported as the mean FWHM and mean FWTM of the LSF.

\section{System spatial resolution}

\section{Method}

A capillary tube line source of internal diameter $0.5 \mathrm{~mm}$ is imaged at the collimator face (parallel-hole collimators) or at the non-magnifying distance (pinhole collimators). The source is imaged in alignment with both the $x$ - and $y$-axes of the detector array.

Measurements are to be repeated at five or more distance intervals up to a distance of $100 \mathrm{~mm}$ from the camera collimator face; in each case the space between the camera face and source should be filled with scattering material such as Perspex or water.

\section{Analysis}

The FWHM and FWTM should be calculated from the LSF profile of each image. These may be averaged for images taken at different orientations at the same distance. These values should be corrected for magnification if applicable, for example, a FWHM of $5 \mathrm{~mm}$ for a line source at a distance resulting in a magnification of 0.2 would have its FWHM resolution recorded as $1 \mathrm{~mm}$, giving resolution in terms of actual object size.

A line of best fit for both distance versus FWHM and distance versus FWTM should be calculated.

\section{Reporting parameters}

FWHM and FWTM should be reported at the collimator face (parallel-hole collimators) or the non-magnifying point (pinhole collimators). The relationship between FWHM and distance in scattering material should be reported along with the range over which this was measured.

\section{Spatial distortion}

\section{Method}

A slit transmission mask is required for intrinsic measurements. The slit should be as narrow as possible; this technique has been successful with a slit of width $2 \mathrm{~mm}$ [27]. The edge should be perpendicular to the mask surface and straight to an accuracy of at least $10 \%$ of the intrinsic resolution of the detector. The mask thickness should at a minimum be sufficient to exclude $99 \%$ of photons, although a thicker mask (e.g. $20 \mathrm{~mm}$ ) would be preferable to exclude divergent photons. The slit should be long enough to extend across the entire detector face. If this is not the case additional images should be taken to ensure full coverage.
The mask is placed as close to the detector as camera design allows. A flood source, or point source at a distance of at least 100 times its diameter (to ensure uniformity), irradiates the mask. The point source should be in line with the centre of the detector.

The spatial non-linearity is assessed by aligning this mask in the two orthogonal (i.e. $x$ and $y$ ) directions of the detector and recording an image in each orientation.

\section{Analysis}

For each image a line of best fit through the centre of the slit image should be calculated. For each row in the image the centre point is compared to its expected position from the line of best fit. The difference between the expected and actual centre positions (deviation) is recorded for each row and for each orientation. These results are then used to calculate the required parameters.

\section{Reporting parameters}

Linearity should be reported as the mean deviation from expected positions, maximum deviation (often reported as absolute linearity) and differential linearity, defined as the standard deviation of these differences.

Intrinsic spatial uniformity

\section{Method}

A flood source, or point source at a distance of at least 100 times its diameter, irradiates the uncollimated detector. The point source should have a maximum diameter of $3 \mathrm{~mm}$; if a planar flood source is used, this should have a coefficient of variation in counts across the flood source of less than $1 \%$.

\section{Analysis}

The mean and standard deviation of counts per pixel over the image should be calculated. Equation (1) should be used to calculate 10 differential uniformity values for each pixel: for the 2, 4, 6, 8 and 10 nearest neighbour pixels in the pixel's row and column [17]. These values are collated and used to produce a frequency histogram for differential uniformity.

\section{Reporting parameters}

Integral uniformity should be reported as the coefficient of variation - the ratio of standard deviation to mean counts reported as a percentage. A frequency histogram of differential uniformity values, a, may be presented or the mean and its dispersion of this can be stated directly. The dispersion of the differential uniformity, $\delta \Omega$, can be calculated using Equation (2):

$\delta \Omega=\left[\frac{\sum_{d=1}^{p} d(a)^{2} n(a)}{\sum_{d=1}^{p} n(a)}\right]^{1 / 2}$

where $n(a)$ is the number of occasions on which a differential uniformity of $d(a)$ is found and $p$ is the number of data classes in the histogram.

\section{Count-rate capability}

\section{Method}

A point source should be placed at a distance of at least 100 times its diameter from the uncollimated detector. Activity within the source should be measured as accurately as possible before imaging starts, as should the distance from source to detector. An identical image should be taken at regular intervals (e.g. every 30 minutes) until the source has decayed to an 
undetectable level. This may be completed in several sessions with different levels of source activity if required in order to cover the full range. If a flood source is used it may be placed as close to the detector face as possible. This should be repeated both with and without $100 \mathrm{~mm}$ Perspex between the radioactive source and the detector surface.

\section{Analysis}

For each image, incident counts at the detector can be calculated taking into account the initial activity, source-detector distance, time of image and the solid angle subtended by the detector at the source. When scattering material is used, this value does not need to be corrected for attenuation. Incident counts are then plotted against recorded counts to produce a count-rate capability curve. Since the incident counts are accumulated over a sufficiently long duration, negligible dead-time count-rate losses will be included. The actual input count-rate where dead-time count-rate losses may be greater can therefore be calculated by decay correcting the later count-rates to those earlier on.

A line of best fit is calculated for the linear portion of the curve. Incident activity at which the measured counts differ from the linear fit by more than $10 \%$ of the expected value is calculated. This should be repeated for results with and without scattering material.

\section{Reporting parameters}

The maximum measured count-rate should be reported. The actual incident count rate at which the measured count rate begins to deviate from the line of best fit by more than $10 \%$ both with and without scattering material should also be reported. This value may be converted back to a point source value for comparison with LFOV systems if required.

\section{Sensitivity}

\section{Method}

A point at a distance of at least 100 times its diameter from the uncollimated detector or flood source with known activity is required. Intrinsic sensitivity is measured with the point source at a distance of at least 100 times its diameter from the uncollimated detector and requires a single image within the detector's countrate capability.

System sensitivity is measured by imaging the point source at the non-magnifying point (pinhole collimators) or the collimator surface (parallel-hole collimators) and then at least five distance intervals up to a distance of $100 \mathrm{~mm}$ from the camera collimator face; in each case the space between the camera face and source should be filled with scattering material such as Perspex or water.

\section{Analysis}

For intrinsic sensitivity incident counts on the detector can be calculated in the same way as described in Section Count-rate capability: analysis. For system sensitivity, a plot of distance against recorded counts is required.

\section{Reporting parameters}

Intrinsic sensitivity is reported as the ratio of detected to counts incident on the detector ( $\mathrm{cps} / \mathrm{MBq}$ incident).

System sensitivity should be reported at the non-magnifying point (pinhole collimators) or the collimator surface (parallelhole collimators) and with $100 \mathrm{~mm}$ distance of scattering material. System sensitivity should be reported in terms of $\mathrm{cps} / \mathrm{MBq}$ within the point source (no conversion to counts incident on detector is required).

\section{Energy resolution}

\section{Method}

A point or flood source within the count-rate capability of the detector is required. At least two radioisotope sources are needed for calibration of the energy spectrum, one of which should be ${ }^{99 \mathrm{~m}} \mathrm{Tc}$. The uncollimated detector is irradiated uniformly and an image taken. This is repeated for each radionuclide used.

\section{Analysis}

Energy channels should be calibrated using ${ }^{99 \mathrm{~m}} \mathrm{Tc}(140.5 \mathrm{keV})$ and the photopeaks of the additional isotopes used. The FWHM of the ${ }^{99 \mathrm{~m}}$ Tc peak is then calculated.

\section{Reporting parameters}

Energy resolution is reported as the FWHM of the ${ }^{99 \mathrm{~m}}$ Tc energy peak as a percentage of $140.5 \mathrm{keV}$.

\section{Conclusions}

This communication adapts the existing protocols, used in clinical environments for assessing the performance of LFOV gamma cameras, for use with SFOV gamma camera systems. These protocols should be developed in the future to take into account specific requirements for SFOV applications, such as detectability of lesions in sentinel lymph node biopsy or image registration in hybrid systems [28]. The described procedures for evaluating SFOV performance parameters provide a more appropriate scheme for characterising SFOV systems. These are applicable to all detector and collimator set-ups, and have been shown to be effective in characterising a SFOV camera [27]. To ensure consistency and comparability the authors recommend their adoption for all SFOV systems.

\section{Declarations}

This work is supported by an STFC CLASP Grant (ST/I00327/4). SLB is in receipt of an STFC studentship. BSB and SLB declare that they have no conflicts of interest. Both JEL and ACP are Directors of a University of Leicester spin out company, Gamma Technologies Ltd.

\section{Acknowledgements}

The authors would like to thank: Elaine Blackshaw, Medical Physics and Clinical Engineering, University Hospitals, Nottingham; Dave Bassford, Oliver Blake, and Adam Bark, Space Research Centre, University of Leicester and David Monk and Helen Hill, Leicester Royal Infirmary, for supporting this work.

\section{References}

[1] Perkins AC, Hardy JG. Intra-operative nuclear medicine in surgical practice. Nucl Med Commun 1996:17:1006-15.

[2] Duch J. Portable gamma cameras: the real value of an additional view in the operating theatre. Eur J Nucl Med Mol Imaging 2011;38:633-5.

[3] Tsuchimochi M. Hayama K. Intraoperative gamma cameras for radioguided surgery: technical characteristics, performance parameters, and clinical applications. Phys Med 2013;29:126-38.

[4] Olcott P, Pratx G, Johnson D, Mittra E, Niederkohr R, Levin CS. Clinical evaluation of a novel intraoperative handheld gamma camera for sentinel lymph node biopsy. Phys Med 2014;30:340-5.

[5] Porras E, Escat B, Benlloch JM, Kadi-Hanifi D, Lopez S, Pavón N, et al. Portable mini gamma camera for medical applications. Nucl Instrum Meth A 2002;486: 186-90.

[6] Ferretti A, Chondrogiannis S, Marcolongo A, Rubello D. Phantom study of a new hand-held $\gamma$-imaging probe for radio-guided surgery. Nucl Med Commun 2013:34:86-90.

[7] Ortega J, Ferrer-Rebolleda J, Cassinello N, Lledo S. Short communication. Potential role of a new hand-held miniature gamma camera in performing 
minimally invasive parathyroidectomy. Eur J Nucl Med Mol Imaging 2007;34: 165-9.

[8] Sánchez F, Benlloch JM, Escat B, Pavón N, Porras E, Kadi-Hanifi D, et al. Design and tests of a portable mini gamma camera. Med Phys 2004;31:1384-97.

[9] De Vree GA, Westra AH, Moody I, van der Have F, Ligtvoet KM, Beekman FJ Photon-counting gamma camera based on an electron-multiplying CCD. IEEE Trans Nucl Sci 2005;52:580-8.

[10] Lees JE, Bassford DJ, Blake OE, Blackshaw PE, Perkins AC. A high resolution small field of view (SFOV) gamma camera: a columnar scintillator coated CCD imager for medical applications. J Instrum 2011;6:1-12.

[11] Fiorini C, Longoni A. Gamma-ray imaging detectors based on silicon drift detectors arrays coupled to a single scintillator. Nucl Instrum Meth A 2003;497:221-5.

[12] Russo P, Curion AS, Mettivier G, Esposito M, Aurilio M, Caraco C, et al. Evaluation of a CdTe semiconductor based compact gamma camera for sentinel lymph node imaging. Med Phys 2011;38:1547.

[13] Gal O, Gmar M, Ivanov O, Lainé F, Lamadie F, Le Goaller C, et al. Development of a portable gamma camera with coded aperture. Nucl Instrum Meth A 2006;563:233-7.

[14] Tsuchimochi M, Sakahara H, Hayama K, Funaki M, Ohno R, Shirahata T, et al. A prototype small CdTe gamma camera for radioguided surgery and other imaging applications. Eur J Nucl Med Mol Imaging 2003;30:1605-14.

[15] Abe A, Takahashi N, Lee J, Oka T, Shizukuishi K, Kikuchi T, Performance evaluation of a hand-held, semiconductor ( $\mathrm{CdZnTe}$ )-based gamma camera. Eur J Nucl Med Mol Imaging 2003;30:805-11.

[16] Chapman J, Hugg J, Vesel J, Bai C, Blevis I, Barrett H, et al. Performance measurement of scintillation cameras. Rosslyn, VA: National Electrical Manufacturers Association; 2007. NEMA NU 1.

[17] Bolster A, Hannan J, Cosgriff PS, Mostaffa AB, Parkin A, Sharp PF, et al. In Bolster A, editor. Report 86 Quality assurance in gamma camera systems. York: Institute of Physics and Engineering in Medicine; 2003, ISBN 1903613 13 2, p. $1-130$
[18] European Council. European directive 97/43/EURATOM regarding the health protection of individuals against the damages of ionizing radiation in relation to medical exposure. OJ L 1997;180:22-7. 9.7.1997, http://www.eur-lex. europa.eu.

[19] Busemann Sokole E, Płachcínska A, Britten A, Lyra Georgosopoulou M, Tindale W, Klett R. Routine quality control recommendations for nuclear medicine instrumentation. Eur J Nucl Med Mol Imaging 2010;37:662-71.

[20] Dondi M, Palm S, Busemann Sokole E, Stodilka RZ, Wegst AV, Zimmerman RE. Quality assurance for SPECT systems. IAEA Hum. Heal. Ser. No. 6. Vienna: IAEA; 2009, ISBN 978920103709 1, p. 1-263.

[21] Busemann Sokole E, Płachcínska A, Britten A. Acceptance testing for nuclear medicine instrumentation. Eur J Nucl Med Mol Imaging 2010;37:672-81.

[22] Vesel J, Petrillo M. Improved gamma camera performance using event positioning method based on distance dependent weighting. IEEE Nucl Sci Conf $\mathrm{R}$ 2005;5:2445-8.

[23] Väyrynen T, Pitkänen U, Kiviniitty K. Methods for measuring the modulation transfer function. Eur J Nucl Med 1980;5:19-22.

[24] Lees JE, Bassford DJ, Blackshaw PE, Perkins AC. Design and use of miniphantoms for high resolution planar gamma cameras. Appl Radiat Isot 2010;68:2448-51.

[25] Metzler SD, Bowsher JE, Smith MF, Jaszczak RJ. Analytic determination of pinhole collimator sensitivity with penetration. IEEE Trans Med Imaging 2001;20:730-41.

[26] Benjegard SA, Sauret V, Bernhardt P, Wangberg B, Ahlman H, ForssellAronsson E. Evaluation of three gamma detectors for intraoperative detection of tumors using In-111 labeled radiopharmaceuticals. J Nucl Med 1999;40: 2094-101.

[27] Bugby SL, Lees JE, Bhatia BS, Perkins AC. Characterisation of a high resolution small field of view portable gamma camera. Phys Med 2013;30:331-9.

[28] Lees JE, Bassford DJ, Blake OE, Blackshaw PE, Perkins AC. A hybrid camera for simultaneous imaging of gamma and optical photons. J Instrum 2012;7:1-11. 